\title{
ALMOST ANALYTIC VECTOR FIELDS IN ALMOST COMPLEX MANIFOLDS
}

\author{
ISUKE SATÔ
}

(Received March 16, 1965)

Introduction. S. Tachibana $([6],[7])^{1)}$ introduced the notion of covariant almost analytic vector field in certain almost Hermitian manifold by generalizing the following fact: "In a compact Kählerian manifold, the inner product of a contravariant analytic vector field and a covariant analytic vector field is constant over the manifold (K. Yano [9])." Further, by several geometors this notion was generalized in general almost complex manifold and to one of almost analytic tensor field.

Let $(M, J)$ and $\left(M^{\prime}, J^{\prime}\right)$ be almost complex manifolds of class $C^{\infty}$ where $J$ and $J^{\prime}$ are almost complex structures on $M$ and $M^{\prime}$ respectively. Let $f$ be a differentiable mapping of $M$ into $M^{\prime}$; the mapping $f$ is called almost analytic if

$$
d f_{P}, \circ J_{P}=J_{f(P)}^{\prime} \circ d f_{P} \quad \text { for } P \in M \text {. }
$$

It is well-known that an almost analytic mapping of a complex manifold into another complex manifold is holomorphic (S. Helgason [1]).

Now, if we adopt a tensor bundle of $M$ as the second manifold $M^{\prime}$ and define a suitable almost complex structure on the tensor bundle of $M$, then almost analytic tensor fields on $M$ can be defined by differentiable cross-section of $M$ in the tensor bundle of $M$. In the present paper we shall consider almost analytic vector fields from this point of view and discuss the relation between the usual almost analytic vector fields and ours. We shall also obtain the generalization of the usual almost analytic vector fields.

1. Contravariant almost analytic vectors. Let $M$ be a $2 n$-dimensional almost complex manifold with structure tensor $\phi$. The set of all tangent vectors of $M$ constitutes, with a natural topology, the so-called tangent bundle of $M$. We denote it by $T(M)$ and the natural projection $T(M) \rightarrow M$ by $\pi$. When $T(M)$ admits an almost complex structure $\Phi$, a differentiable cross-section $f$ is called contravariant almost analytic vector field with recpect to $(\phi, \Phi)$ over $M$ if

1) See the bibliography at the end of the paper. 


$$
d f_{P} \circ \varphi_{P}=\Phi_{f(P)} \circ d f_{P} \quad \text { for } \quad P \in M \text {. }
$$

Let $x^{i 2)}$ be local coordinates in a neighborhood $U$ of a point $P$ of $M$, then a tangent vector $y$ at $P$, which is an element of $T(M)$, is expressible in the form $\left(x^{i}, y^{i}\right)$ where $y^{i}$ s are components of $y$ with respect to the natural frame $\partial / \partial x^{i}$. We may consider $\left(x^{i}, y^{i}\right)$ local coordinates in a neighborhood $\pi^{-1}(U)=U \times E^{n}$ of $T(M)$. To a transformation of local coordinates of $M$

$$
x^{i^{\prime}}=x^{i^{\prime}}\left(x^{1}, x^{2}, \cdots, x^{2 n}\right)
$$

there corresponds in $T(M)$ the coordinate transformation

$$
\left\{\begin{array}{l}
x^{i^{\prime}}=x^{i^{\prime}}\left(x^{1}, x^{2}, \cdots, x^{2 n}\right), \\
y^{i^{\prime}}=\left(\partial_{r} x^{i^{\prime}}\right) y^{r} .
\end{array}\right.
$$

If we put

$$
x^{i}=y^{i} \quad x^{i^{\prime}}=y^{i \prime},{ }^{3)}
$$

then we may write (1.2) as

$$
x^{I^{\prime}}=x^{I^{\prime}}\left(x^{1}, x^{2}, \cdots, x^{4 n}\right)
$$

Let $\phi$ be a tensor field of type $(1,1)$ on $M$ and $\phi_{j}{ }^{i}$ be its components with respect to a coordinate neighborhood $U\left(x^{i}\right)$. If we put

$$
\begin{cases}\Phi_{j}{ }^{i}=\boldsymbol{\phi}_{j}{ }^{i}, & \Phi_{j}{ }^{i}=y^{r} \partial_{r} \boldsymbol{\varphi}_{j}{ }^{i}, \\ \Phi_{j}{ }^{i}=0, & \Phi_{j}{ }^{i}=\boldsymbol{\phi}_{j}{ }^{i}\end{cases}
$$

then we have a tensor field $\Phi$ of type $(1,1)$ on $T(M)$ whose components are $\Phi_{J}{ }^{I}$ with respect to the coordinate neighborhood $\pi^{-1}(U)\left(x^{i}, y^{i}\right)$. $\Phi$ is called the extension of $\varphi$ (S. Sasaki [4]). We can easily see that if $\varphi_{j}{ }^{i}$ is an almost complex structure, so is also $\Phi_{J}{ }^{I}$.

Now, cross-section $f$ can be locally expressed by

$$
\bar{x}^{I}=\bar{x}^{I}\left(x^{1}, x^{2}, \cdots, x^{2 n}\right),
$$

namely

2) Indices $i, j, k ; a, b, c ; r, s, t$ run over the range $1,2, \cdots, 2 n$.

3) We put $\bar{i}=2 n+i, \bar{i}^{\prime}=2 n+i^{\prime}$.

4) $I, J, K=1,2, \cdots, 4 n$, 


$$
\left\{\begin{array}{l}
\bar{x}^{i}=x^{i}, \\
y^{i}=u^{i}\left(x^{1}, x^{2}, \cdots, x^{2 n}\right) .
\end{array}\right.
$$

By virtue of (1.1), (1.4) and (1.5), locally the condition that $f$ be almost analytic is

$$
\varphi_{j}^{r} \partial_{r} \bar{x}^{I}=\Phi_{R}^{I} \partial_{j} \bar{x}^{R}
$$

In the case $I=i$ we get the identity $\varphi_{j}{ }^{i}=\varphi_{j}{ }^{i}$ and in the case $I=\bar{i}$

or

$$
\boldsymbol{\varphi}_{j}{ }^{r} \partial_{r} u^{i}=u^{r} \partial_{r} \varphi_{j}{ }^{i}+\varphi_{r}{ }^{i} \partial_{j} u^{r}
$$

$$
\Re(u) \varphi_{j}{ }^{i}=0 .
$$

As the last equation is nothing but the defining equation of usual contravariant almost analytic vector field in the almost complex manifold $M$, our contravariant almost analytic vector field coincides with the usual one. Consequently, the usual contravariant almost analytic vector field is regarded as the contravariant almost analytic vector field with respect to $\phi$ and its extension $\Phi$ which is considered from the point of $\phi$ only. We denote it by the contravariant almost analytic vector field with respect to $\phi$.

THEOREM 1.1. In order that an extension $\bar{u}^{I}=\left(u^{i}, y^{r} \partial_{r} u^{i}\right)$ of a contravariant vector field $u^{i}$ of $M$ be contravariant almost analytic with respect to $\Phi$ on $T(M)$, it is necessary and sufficient that $u^{i}$ itself is contravariant almost analytic with respect to $\phi$ on $M$.

PROOF. Let $u^{i}$ be contravariant almost analytic on $M$, i.e. $\mathfrak{L}(u) \varphi_{j}{ }^{i}=0$. After some calculations we get

$$
\begin{aligned}
& \mathfrak{L}(\bar{u}) \Phi_{j}{ }^{i}=\mathfrak{l}(\bar{u}) \Phi_{j}{ }^{i}=\mathfrak{L}(u) \varphi_{j}{ }^{i}=0, \\
& \mathfrak{L}(\bar{u}) \Phi_{j}{ }^{i}=0, \quad \mathfrak{l}(\bar{u}) \Phi_{j}{ }^{\bar{i}}=y^{r} \partial_{r}\left(\mathfrak{L}(u){\varphi_{j}}^{i}\right)=0,
\end{aligned}
$$

from which we have $\mathfrak{L}(\bar{u}) \Phi_{J}^{I}=0$ i.e. $\bar{u}$ is contravariant almost analytic on $T(M)$. Converse is evident.

Next, we shall introduce an almost complex structure on $T(M)$ different from $\Phi_{J}^{I}$. Let $\Gamma_{j i}^{h}$ be an affine connection on $M$. If we define $\widetilde{\Phi}_{J}^{I}$ by

$$
\begin{cases}\widetilde{\Phi}_{j}{ }^{i}=\Gamma_{j}{ }^{i}, & \widetilde{\Phi}_{j}{ }^{\bar{i}}=-\left(\delta_{j}{ }^{i}+\Gamma_{j}{ }^{r} \Gamma_{r}{ }^{i}\right), \\ \widetilde{\Phi}_{\bar{j}}{ }^{i}=\delta_{j}{ }^{j}, & \widetilde{\Phi}_{\bar{j}}{ }^{i}=-\Gamma_{j}{ }^{i}\end{cases}
$$


where $\Gamma_{j}{ }^{i}=\Gamma_{j r}{ }^{i} y^{r}$, then it is an almost complex structure on $T(M)$ (C. T. Hsu $[2])$. By virtue of $\widetilde{\Phi}$, we can find the contravariant almost analytic vector field with respect to $(\varphi, \widetilde{\Phi})$. In the same way as before, by (1.1), (1.5) and (1.7), we have

$$
\phi_{j}{ }^{r} \partial_{r} x^{l}=\tilde{\Phi}_{l}{ }^{I} \partial_{j} \bar{x}^{r}
$$

Putting $I=i$ and $I=\bar{i}$, we have

$$
\varphi_{j}^{i}=\widetilde{\nabla}_{j} u^{i}
$$

and

$$
\phi_{j}{ }^{r} \partial_{r} u^{i}=-\left(\delta_{j}{ }^{i}+\Gamma_{j}{ }^{r} \Gamma_{r}{ }^{i}+\Gamma_{r}{ }^{i} \partial_{j} u^{r}\right),
$$

where $\widetilde{\nabla}_{j}$ denotes the operator of covariant derivative with respect to the affine connection $\Gamma_{j i}{ }^{h}$. However, the last equation is identically satisfied by (1.8). In fact, we have the following

$$
\begin{aligned}
& \phi_{j}{ }^{r} \partial_{r} u^{i}+\delta_{j}{ }^{i}+\Gamma_{j}{ }^{r} \Gamma_{r}{ }^{i}+\Gamma_{r}{ }^{i} \partial_{j} u^{r} \\
& \quad=\phi_{j}{ }^{r}\left(\phi_{r}{ }^{i}-\Gamma_{r}{ }^{i}\right)+\delta_{j}{ }^{i}+\Gamma_{j}{ }^{r} \Gamma_{r}{ }^{i}+\Gamma_{r}{ }^{i}\left(\phi_{j}{ }^{r}-\Gamma_{j}{ }^{r}\right)=0
\end{aligned}
$$

Thus contravariant almost analytic vector field with respect to $(\phi, \widetilde{\Phi})$ is given by (1.8). In general, contravariant almost analytic vector field satisfying (1.8) does not satisfy (1.6) i.e. not contravariant almost analytic in the sense of the usual one. Therefore, we shall call the contravariant almost analytic vector field with respect to $(\varphi, \Phi)$ and $(\varphi, \widetilde{\Phi})$ as the contravariant almost analytic vector fields of the first kind and the second kind of $M$ respectively.

In particular, if $M$ is almost Hermitian, (1.8) may be written as

$$
\varphi_{j}{ }^{i}=\nabla_{j} u^{i}
$$

or

$$
\nabla_{j} u_{i}=\boldsymbol{\varphi}_{j i} \quad\left(\boldsymbol{\phi}_{j i}=\boldsymbol{\phi}_{j}{ }^{r} g_{r i}\right)
$$

where $\nabla_{j}$ denotes the operator of covariant derivative with respect to the Christoffel's symbols $\left\{\begin{array}{c}h \\ j i\end{array}\right\}$. From (1.9) we see that contravariant almost analytic vector field of the second kind $u^{i}$ is a Killing vector field since $\phi_{j i}$ is skew-symmetric. Therefore almost Hermitian manifolds admitting contravariant almost analytic vector field of the second kind coincides with certain almost Kählerian manifolds introduced by Y. Mutô (Y. Mutô [3]). Thus almost 
Hermitian manifold with contravariant almost analytic vector field of the second kind becomes necessarily almost Kählerian one and contravariant almost analytic vector field of the second kind the one of the first kind (Y. Muto [3]).

THEOREM 1.2. In a compact almost Hermitian manifold $M$ there does not exist any contravariant almost analytic vector field of the second kind.

PROOF. Let $u^{i}$ be contravariant almost analytic vector field of the second kind. Then we get

$$
\left(\nabla^{j} u^{i}\right) \nabla_{j} u_{i}=\nabla_{j}\left(\left(\nabla^{j} u^{i}\right) u_{i}\right)-\left(\nabla_{j} \nabla^{j} u^{i}\right) u_{i}=\nabla_{j}\left(\left(\nabla^{j} u^{i}\right) u_{i}\right)
$$

because $\nabla_{j} \nabla^{j} u^{i}=\nabla_{j} \varphi^{j i}=0$.

By integration it follows that

$$
\int_{M}\left(\nabla^{j} u^{i}\right) \nabla_{j} u_{i} d \sigma^{=}=\int_{M} \nabla_{j}\left(\left(\nabla^{j} u^{i}\right) u_{i}\right) d \sigma=0
$$

where $d \sigma$ is the volume element of $M$. From the last equation we have $\nabla_{j} u_{i}=0$ i.e. $\varphi_{j i}=0$. This is a contradiction.

Q.E.D.

THEOREM 1.3. In an almost Hermitian manifold with parallel Rcici tensor field $R_{j i}\left(R_{j i} \neq 0\right)$, there does not exist any contravariant almost analytic vector field of the second kind.

PROOF. Let $u^{i}$ be contravariant almost analytic vector field of the second kind. As $u^{i}$ is a Killing vector field we get

$$
\nabla^{r} \nabla_{r} u^{i}+R_{r}^{i} u^{r}=0 \text {. }
$$

By virtue of (1.9) and $\nabla^{r} \boldsymbol{\varphi}_{r}{ }^{i}=0$ we have $R_{i r} u^{r}=0$. Operating $\nabla_{k}$ to $R_{i r} u^{r}=0$ and making use of $\nabla_{k} R_{i r}=0$, we have $R_{i r} \nabla_{k} u^{r}=0$. Again by (1.9), we get $R_{i r} \varphi_{k}{ }^{r}=0$ i.e. $R_{i j}=0$. This is a contradiction.

THEOREM 1.4. If an almost Hermitian manifold admitting a contraavriant almost analytic vector field of the second kind is flat, then the almost complex structure is integrable.

PROOF. It follows immediately by

$$
N_{j i}{ }^{h}=2 \phi_{j}{ }^{s} R_{s i r}{ }^{h} u^{r} .
$$


2. Cotangent bundles. Let $M$ be an $n$-dimensional differentiable manifold of class $C^{\infty}$. The set of all non-zero covectors of $M$ constitutes, with natural topology, the so-called cotangent bundle of $M$. We denote it by " $T(M)$ and the natural projection " $T(M) \rightarrow M$ by $\pi$. Let $x^{i}$ be local coordinates in a neighborhood $U$ of a point of $M$, then a cotangent vector $y$ at $P$ which is an element of ${ }^{c} T(M)$ is expressible in the form $\left(x^{i}, y_{i}\right)^{5)}$ where $y_{i}$ are components of $y$ with respect to the natural coframe $d x^{i}$. We may consider $\left(x^{i}, y_{i}\right)$ local coordinates in a neighborhood $\pi^{-1}(U)=U \times E^{n}$ of ${ }^{c} T(M)$.

To a transformation of local coordinates of $M$

$$
x^{i^{\prime}}=x^{i^{\prime}}\left(x^{1}, x^{2}, \cdots, x^{n}\right),
$$

there corresponds in ${ }^{C} T(M)$ the coordinate transformation

$$
\left\{\begin{array}{l}
x^{i^{\prime}}=x^{i^{\prime}}\left(x^{1}, x^{2}, \cdots, x^{n}\right) \\
y_{i^{\prime}}=\left(\partial_{i^{\prime}} x^{i}\right) y_{i} .
\end{array}\right.
$$

If we put

$$
x^{\bar{i}}=y_{i} \quad x^{\bar{i}^{\prime}}=y_{i^{\prime}},
$$

then we may write $(2.1)$ as

$$
x^{I^{\prime}}=x^{I^{\prime}}\left(x^{1}, x^{2}, \cdots, x^{2 n}\right) .
$$

So we may define tensors of ${ }^{\prime} T(M)$ by making use of a matrix $\left(\frac{\partial x^{I^{\prime}}}{\partial x^{I}}\right)$ i.e.

$$
\left(\begin{array}{lc}
\frac{\partial x^{i^{\prime}}}{\partial x^{i}} & 0 \\
\frac{\partial}{\partial x^{i}}\left(\frac{\partial x^{r}}{\partial x^{i^{\prime}}}\right) y_{r} & \frac{\partial x^{i}}{\partial x^{i^{\prime}}}
\end{array}\right),
$$

its transposed matrix and their Kronecker products. Making use of (2.3), we have after some complicated calculations, the following lemmas.

LEMMA 1. Let $t$ be a tensor field of type $(1,1)$ on $M$ and $t_{j}{ }^{\prime}$ 's are its components with respect to a coordinate neighborhood $U\left(x^{i}\right)$. If we put

5) In this section, the indices run over as follows,

$$
i, j, k=1,2, \cdots, n ; \bar{i}=n+i, \bar{i}^{\prime}=n+i^{\prime} ; I, J, K=1,2, \cdots, 2 n \text {. }
$$




$$
\begin{cases}T_{j}{ }^{i}=t_{j}{ }^{i}, & T_{j}{ }^{\bar{i}}=\left(\partial_{j} t_{i}{ }^{r}-\partial_{i} t_{j}{ }^{r}\right) y_{r}, \\ T_{\bar{j}}{ }^{i}=0, & T_{\bar{j}}{ }^{\bar{i}}=t_{i}{ }^{j},\end{cases}
$$

then we get a tensor field $T$ of type $(1,1)$ on ' $T(M)$ whose components are $T_{J}{ }^{I}$ with respect to the coordinate neighborhood $\pi^{-1}(U)\left(x^{i}, y_{i}\right)$.

LEMMA 2. Let $M$ be an almost complex manifold with the structure tensor $\phi$ and $\phi_{j}{ }^{i}$ 's are its components with respect to a coordinate neighborhood $U\left(x^{i}\right)$. If we put

$$
\begin{cases}F_{j}{ }^{i}=\boldsymbol{\phi}_{j}{ }^{i}, & F_{j}^{\bar{i}}=\frac{1}{2}\left\{\partial_{j} \boldsymbol{\varphi}_{i}{ }^{r}-\partial_{i} \boldsymbol{\varphi}_{j}{ }^{r}+\boldsymbol{\varphi}_{j}{ }^{t} \boldsymbol{\varphi}_{i}{ }^{s}\left(\partial_{t} \boldsymbol{\varphi}_{s}{ }^{r}-\partial_{s} \boldsymbol{\varphi}_{l}{ }^{r}\right)\right\} y_{r}, \\ F_{j}{ }^{i}=0, & F_{j}{ }^{\bar{i}}=\boldsymbol{\varphi}_{i}{ }^{j},\end{cases}
$$

then we get a tensor field $F$ of type $(1,1)$ on ${ }^{C} T(M)$ whose components are $F_{. I}^{I}$ with respect to the coordinate neighborhood $\pi^{-1}(U)\left(x^{i}, y_{i}\right)$. Moreorer it is an almost complex structure on ' $T(M)$.

Next, take a homogeneous contact form $\eta_{y}=y_{i} d x^{i}$ of $M$ which is a global 1 -form over ${ }^{C} T(M)$ and consider the exterior differential $d \eta$ of the contact form $\eta$ (S. Sasaki [5]). In every neighborhood, it is given by

$$
d \eta=\frac{1}{2} \widetilde{F}_{I J} d x^{I} \wedge d x^{I}
$$

where we have put

$$
\widetilde{F}_{I J}=\partial_{I} \eta_{J}-\partial_{J} \eta_{I} \quad \eta_{I}=\left(0, y_{i}\right) \text {. }
$$

We can easily see that $\left(\widetilde{F}_{I . J}\right)$ has the following numerical components:

$$
\widetilde{F}_{i j}=0 \quad \widetilde{F}_{i j}=-\delta_{i}{ }^{j} \quad \widetilde{F}_{i j}=\delta_{j}{ }^{i} \quad \widetilde{F}_{i j}=0 .
$$

which is called the fundamental null system of ${ }^{\circ} T(M)$.

Now, let $g$ be the fundamental metric tensor of $M$ and $g_{j k}$ 's are the components of $g$ with respect to a coordinate neighborhood $U\left(x^{i}\right)$ in $M$. We shall define a line element in a coordinate neighborhood $\pi^{-1}(U)\left(x^{i}, y_{i}\right)$ of ${ }^{c} T(M)$ by

$$
d \sigma^{2}=g_{j k}(x) d x^{j} d x^{k}+g^{j k}(x) D y_{j} D y_{k},
$$


where $D y_{j}$ means the covariant differential of $y_{j}$, i.e.

$$
D y_{j}=d y_{j}-\left\{\begin{array}{c}
i \\
j k
\end{array}\right\} y_{i} d x^{k}
$$

$\left\{\begin{array}{c}i \\ j k\end{array}\right\}$ being Christoffel's symbols. It is clear that the right hand side of (2.8) is invariant under transformations of ${ }^{c} T(M)$ and positive definite. In the following we shall consider the cotangent bunble ${ }^{C} T(M)$ as a Riemannian manifold endowed with the metric (2.8).

The components of the fundamental metric tensor of ${ }^{c} T(M)$ with respect to the coordinate neighborhood $\pi^{-1}(U)\left(x^{i}, y_{i}\right)$ can be obtained by putting (2.8) in the form

$$
d \sigma^{2}=G_{J K} d x^{J} d x^{K} \quad\left(d x^{\bar{j}}=d y_{j}\right) .
$$

We can easily see that

$$
G_{j k}=g_{j k}+\gamma_{j}{ }^{i} \gamma_{i k}, \quad G_{j \bar{k}}=-\gamma_{j}{ }^{k}, \quad G_{\bar{j} \bar{k}}=g^{j k},
$$

where we have put

$$
\gamma_{j k}=\left\{\begin{array}{c}
i \\
j k
\end{array}\right\} y_{i}
$$

We can explicitly give the contravariant components of the fundamental metric tensor of ${ }^{C} T(M)$. This is easily done by solving the equation

$$
G_{J K} G^{K I}=\delta_{J}^{I}
$$

thinking $G^{K I}$ as unknown variables. The results is as follows :

$$
G^{j k}=g^{j k}, \quad G^{j \bar{k}}=\gamma_{k}{ }^{j}, \quad G^{\bar{j} \bar{k}}=g_{j k}+\gamma_{j}{ }^{i} \gamma_{i k} .
$$

Thus we have

THEOREM 2.1. Let ${ }^{C} T(M)$ be a cotangent bundle of Riemannian manifold $M$ and $\widetilde{F_{I J}}$ and $G_{I J}$ the components of the fundamental null-system and the fundamental metric tensor of ${ }^{C} T(M)$ respectively. If we define $\widetilde{F}_{J}^{I}$ by

$$
\widetilde{F}_{J}^{I}=\widetilde{F}_{J K} G^{K I}
$$

then it is an almost complex structure on ${ }^{C} T(M)$. Moreover $G_{I J}$ is an almost 
Hermitian metric with respect to the almost complex structure $\widetilde{F}_{J}^{I}$ and the almost Hermitian manifold with the structure tensors $\widetilde{F}_{J}^{I}$ and $G_{I J}$ becomes necessarily an almost Kählerian manifold. (P. Tondeur [8]).

We can easily see that $\widetilde{F}_{J}^{I}$ has the following components :

$$
\widetilde{F}_{j}^{i}=-\gamma_{j}{ }^{i}, \quad \widetilde{F}_{\bar{j}}{ }^{i}=g^{i j}, \quad \widetilde{F}_{j}{ }^{\bar{i}}=-\left(g_{i j}+\gamma_{i}{ }^{k} \gamma_{k j}\right), \quad \widetilde{F}_{\bar{j}}{ }^{\bar{i}}=\gamma_{i}{ }^{j} .
$$

THEOREM 2.2. In order that an infinitesimal transformation of Riemannian manifold $M$ is an infinitesimal isometry, it is necessary and sufficient that its extension in ${ }^{\circ} T(M)$ is a contravariant almost analytic vector field of the first kind.

PROOF. By extension $\xi^{I}$ in ${ }^{C} T(M)$ of a vector field $\xi^{i}$ on $M$ we understand a vector field whose components are $\left(-\xi^{i}, y_{r} \partial_{i} \xi^{r}\right)$. In fact, we can easily verify that the extension is a vector field in ${ }^{C} T(M)$. After some complicated calculations we have the following equations:

$$
\begin{aligned}
& \mathfrak{L}(\bar{\xi}) \widetilde{F}_{j}^{i}=\left\{g^{i s}\left(\nabla_{s} \nabla_{j} \xi^{r}+R_{t s j}{ }^{r} \xi^{l}\right)-\left\{\begin{array}{c}
r \\
j s
\end{array}\right\}\left(\nabla^{s} \xi^{i}+\nabla^{i} \xi^{s}\right)\right\} y_{r}, \\
& \mathfrak{Q}(\bar{\xi}) \widetilde{F}_{\bar{j}}^{i}=\nabla^{j} \xi^{i}+\nabla^{i} \xi^{j}, \\
& \Re(\bar{\xi}) \widetilde{F}_{j}^{\bar{i}}=\left\{\boldsymbol{\gamma}_{i}^{s}\left(\nabla_{j} \nabla_{s} \xi^{r}+R_{t j s}^{r}{ }^{r} \xi^{t}\right)+\gamma_{j}^{s}\left(\nabla_{i} \nabla_{s} \xi^{r}+R_{t i s}^{r} \xi^{l}\right)\right\} y_{r} \\
& -\gamma_{j r} \gamma_{i s}\left(\nabla^{r} \xi^{s}+\nabla^{s} \xi^{r}\right)+\left(\nabla_{j} \xi_{i}+\nabla_{i} \xi_{j}\right) \\
& \Omega(\bar{\xi}) \widetilde{F}_{j}^{\bar{i}}=\left\{-g^{j s}\left(\nabla_{s} \nabla_{i} \xi^{r}+R_{t s i}{ }^{r} \xi^{t}\right)+\left\{\begin{array}{c}
r \\
i s
\end{array}\right\}\left(\nabla^{j} \xi^{s}+\nabla^{s} \xi^{j}\right)\right\} y_{r} .
\end{aligned}
$$

From these equations the theorem follows.

3. Covariant almost analytic vectors. Let $M$ be a $2 n$-dimensional almost complex manifold with the structure tensor $\varphi$. When the cotangent bundle ${ }^{\prime} T(M)$ of $M$ admits an almost complex structure $F$, a differentiable crosssection $f$ is called covariant almost analytic vector field with respect to $(\varphi, F)$ over $M$, if

$$
d f_{P} \circ \phi_{P}=F_{f(P)} \circ d f_{P} \quad \text { for } \quad P \in M \text {. }
$$

Now, a cross-section $f$ can be locally expressed by

$$
\bar{x}^{I}=\bar{x}^{I}\left(x^{1}, x^{2}, \cdots, x^{2 n}\right)
$$


or

$$
\left\{\begin{array}{l}
\bar{x}^{i}=x^{i} \\
\bar{y}_{i}=w_{i}\left(x^{1}, x^{2}, \cdots, x^{2 n}\right) .
\end{array}\right.
$$

Since $\phi$ and $F$ defined in Lemma 2 in $\S 2$ are both almost complex structure on $M$ and " $T(M)$ respectively, by the same way as the contravariant case, the condition that the vector field $w_{i}$ is covariant almost analytic with respect to $(\varphi, F)$ is given by

$$
\boldsymbol{\varphi}_{j}{ }^{r} \partial_{r} w_{i}=\frac{1}{2}\left\{\partial_{j} \boldsymbol{\varphi}_{i}{ }^{r}-\partial_{i} \boldsymbol{\varphi}_{j}{ }^{r}+\boldsymbol{\varphi}_{j}{ }^{b} \boldsymbol{\varphi}_{i}{ }^{a}\left(\partial_{l} \boldsymbol{\varphi}_{a}{ }^{r}-\partial_{a} \boldsymbol{\varphi}_{b}{ }^{r}\right)\right\} y_{r}+\boldsymbol{\varphi}_{i}{ }^{r} \partial_{j} w_{r}
$$

which is equivalent to the following equation

$$
\partial_{j}\left(\boldsymbol{\phi}_{i}{ }^{r} \mathfrak{w}_{r}\right)=w_{r} \partial_{i} \boldsymbol{\phi}_{j}{ }^{r}+\boldsymbol{\phi}_{j}{ }^{r} \partial_{r} w_{i}+\frac{1}{2} N_{j i}{ }^{s} \boldsymbol{\phi}_{s}{ }^{r} w_{r}
$$

where $N_{j i}{ }^{s}$ is Nijenhuis' tensor of the structure tensor $\varphi$.

If $M$ is almost Hermitian, then (3.4) may be written as

$$
\nabla_{j}\left(\boldsymbol{\phi}_{i}{ }^{r} w_{r}\right)=w_{r} \nabla_{i}{\boldsymbol{\varphi}_{j}}^{r}+{\boldsymbol{\varphi}_{j}}^{r} \nabla_{r} w_{i}+\frac{1}{2} N_{j i}{ }^{s} \boldsymbol{\varphi}_{s}^{r} w_{r}
$$

From (3.4) we have

$$
\left(\partial_{j}{\varphi_{i}}^{r}-\partial_{i} \phi_{j}{ }^{r}\right) w_{r}=\phi_{j}{ }^{r} \partial_{r} w_{i}-\phi_{i}{ }^{r} \partial_{j} w_{r}+\frac{1}{2} N_{j i}{ }^{s} \phi_{s}{ }^{r} w_{r}
$$

Making use of (3.6), we get

$$
\begin{aligned}
N_{j i}{ }^{h} w_{h} & =\left\{{\boldsymbol{\varphi}_{j}}^{a}\left(\partial_{a} \boldsymbol{\varphi}_{i}{ }^{h}-\partial_{i} \boldsymbol{\varphi}_{a}{ }^{h}\right)-\boldsymbol{\varphi}_{i}{ }^{a}\left(\partial_{a} \boldsymbol{\varphi}_{j}{ }^{h}-\partial_{j} \boldsymbol{\varphi}_{a}{ }^{h}\right)\right\} w_{h} \\
& =-2^{*} O_{j i}^{s r}\left(\partial_{s} w_{r}-\partial_{r} w_{s}\right)+N_{j i}{ }^{h} \mathfrak{w}_{h}
\end{aligned}
$$

i.e.

$$
* O_{j i}^{s \prime}\left(\partial_{s} w_{r}-\partial_{r} w_{s}\right)=0
$$

Thus we have the following

THEOREM 3.1. In an almost complex manifold $M$ with the structure tensor $\phi$ the tensor field $\partial_{[j} w_{i]}$ is pure for a covariant almost analytic vector field $w$. 
REMARK. In the equation (3.4) if we take away the third term in the right hand side, then we get the equation defining the usual covariant almost analytic vector field in $M$. (S. Tachibana [6], [7]).

If in the Lemma 1 of $\$ 2$ we use an almost complex structure $\varphi_{j}{ }^{i}$ instead of general tensor field $t_{j}{ }^{i}$, then we have the tensor $E_{J}{ }^{I}$ defined by

$$
\begin{cases}E_{j}{ }^{i}=\boldsymbol{\varphi}_{j}{ }^{i}, & E_{j}{ }^{\bar{i}}=\left(\partial_{j} \boldsymbol{\varphi}_{i}{ }^{r}-\partial_{i} \boldsymbol{\varphi}_{j}{ }^{r}\right) y_{r}, \\ E_{j}{ }^{i}=0, & E_{j}{ }^{\bar{i}}=\boldsymbol{\varphi}_{i}{ }^{j} .\end{cases}
$$

As we have

$$
E_{j}^{R} E_{R}^{i}=-\delta_{j}{ }^{i}, \quad E_{j}^{n} E_{n}{ }^{i}=0, \quad E_{j}^{R} E_{R}^{\bar{i}}=N_{j i}^{h} y_{h}, \quad E_{\bar{j}}{ }^{n} E_{R}^{\bar{i}}=-\delta_{j}^{i} .
$$

the tensor field $E_{J}{ }^{I}$ is not always an almost complex structure of ${ }^{c} T(M)$. However, if we use the tensor field $E_{J}{ }^{I}$ in the place of the almost complex structure $F_{I}^{I}$ in $(3.1)$, then we have

$$
\partial_{j}\left(\boldsymbol{\phi}_{i}{ }^{r} w_{r}\right)=w_{r} \partial_{i} \boldsymbol{\phi}_{j}{ }^{r}+\boldsymbol{\phi}_{j}{ }^{r} \partial_{r} w_{i}
$$

which is the equation defining the usual covariant almost analytic vector field. Although this shows that the usual covariant almost analytic vector field is not appropriate to call covariant almost analytic in the sense of our standpoint, we can easily find, by comparing the equations (3.4) and (3.7), that the usual one is a special covariant almost analytic one with the property $N_{j i}{ }^{h} w_{h}=0$, since it is well-known that the usual covariant almost analytic vector field has the identity $N_{j i}{ }^{h} w_{h}=0$. (K. Yano and M. Ako [10]).

Next, since we have seen that the tensor field $\widetilde{F}_{J}{ }^{r}$ is an almost complex structure in ${ }^{C} T(M)$, we can define covariant almost analytic vector field with respect to $(\varphi, \widetilde{F})$. In the same way as before, we get, in an almost Hermitian manifold with the structure tensor $(G, \widetilde{F})$, the relation

$$
\nabla_{j} w_{i}=\varphi_{j i}
$$

by (2.14), (3.1) and (3.2). From (3.8) we can see that

(i) $w$ is a Killing vector field,

(ii) $w$ satisfying (3.8) does not satisfy (3.4),

(iii) $w$ is also contravariant almost analytic vector field of the second kind by virtue of (1.9).

Because of the property (ii) above, we shall call the covariant almost analytic vector fields with respect to $(\varphi, F)$ and $(\varphi, \widetilde{F})$ as the covariant almost analytic vector fields of the first kind and the second kind of $M$ respectively. 
THEOREM 3.2. In an almost Hermitian manifold, let $u^{i}$ be a contravariant almost analytic vector field of the second kind. Then $g_{j i} u^{i}$ is covariant almost analytic one of the second kind.

4. Properties of covariant almost analytic vectors in $* 0$-space. By $* O$-space we mean a $2 n$-dimensional differentiable manifold $M$ with a fixed almost Hermitian structure $\left(\varphi_{j}{ }^{i}, g_{j i}\right)$ such that

$$
* O_{j i}^{j a} \nabla_{b} \boldsymbol{\varphi}_{a h}=0
$$

where $\boldsymbol{\phi}_{j i}=\boldsymbol{\varphi}_{j}{ }^{r} g_{r i}$. It is well-known that an almost Kählerian manifold and a $K$-space are ${ }^{*} O$-spaces. In the present section by covariant almost analytic vector field we shall mean covariant almost analytic one of the first kind.

In an $* O$-space, the following identities

$$
\nabla^{r} \boldsymbol{\varphi}_{r i}=0
$$

and

$$
N_{j i l h}=2 \boldsymbol{\varphi}_{j}^{r}\left(\nabla_{r} \boldsymbol{\varphi}_{i h}-\nabla_{i} \boldsymbol{\varphi}_{r h l}\right)
$$

hold good. On the other hand, in a general almost Hermitian manifold

$$
* O_{i h}^{b a} \nabla_{j} \varphi_{b a}=0
$$

is always valid.

Now, we shall deform the equation (3.5) which gives the definition of a covariant almost analytic vector field. From (3.5), we have

$$
w^{r} \nabla_{i} \boldsymbol{\varphi}_{j r}-w^{r} \nabla_{j} \boldsymbol{\phi}_{i r}+\frac{1}{2} N_{j i}{ }^{s} \boldsymbol{\varphi}_{s}^{r} w_{r}+\boldsymbol{\phi}_{j}^{r} \nabla_{r} w_{i}-\boldsymbol{\phi}_{i}{ }^{r} \nabla_{j} w_{r}=0
$$

On taking account of (4.1), (4.3) and (4.4), (4.5) becomes the following form

$$
\phi_{j}^{r} \nabla_{r} w_{i}-\phi_{i}^{r} \nabla_{j} w_{r}=0 \text {. }
$$

Hence, we have the following

THEOREM 4.1. A necessary and sufficient condition for a vector field $w_{i}$ in an ${ }^{*} O$-space to be a covariant almost analytic one of the first kind is that

$$
\phi_{j}^{r} \nabla_{r} w_{i}-\phi_{i}^{r} \nabla_{j} w_{r}=0 \text {. }
$$


We notice that the equation (4.6) is the same form as the one in Kählerian manifold (K. Yano [9]).

Next, applying $\boldsymbol{\varphi}_{k}{ }^{i} \nabla^{j}$ to (4.6), we have

$$
\nabla^{j} \nabla_{j} w_{k}-R^{*}{ }_{k r} w^{r}-\boldsymbol{\varphi}_{k}{ }^{i}\left(\nabla^{j} \varphi_{i}{ }^{r}\right) \nabla_{j} w_{r}=0,
$$

where we have put

$$
R_{k r}^{*}=\frac{1}{2} \phi^{p q} R_{p q s r} \boldsymbol{\varphi}_{k}{ }^{s}
$$

and

$$
\boldsymbol{\phi}^{p q l}=\boldsymbol{\varphi}_{s}^{q} g^{s p}
$$

Let $w_{k}$ be a vector field and define $N(w)_{k}$ by the equation

$$
N(w)_{k}=\boldsymbol{\phi}_{k}^{r}\left(\nabla_{j} \boldsymbol{\varphi}_{i r}\right) \nabla^{j} w^{i},
$$

where $\nabla^{i} w^{i}=g^{j r} \nabla_{r} w^{i}$. On taking account of (4.8), (4.7) may be written as

$$
\nabla^{j} \nabla_{j} w_{k}-R^{*}{ }_{k r} w^{r}+N(w)_{k}=0
$$

Now, let $w_{k}$ be a vector field and introduce a tensor field

$$
t(w)_{j k}=T(w)_{j i} g^{i l} \boldsymbol{\varphi}_{l k},
$$

where

$$
T(w)_{j i}=\varphi_{j}^{r} \nabla_{r} w_{i}-\varphi_{i}^{r} \nabla_{j} w_{r}
$$

For simplicity, we shall denote $t_{j i}$ instead of $t(w)_{j i}$ in the following. $t_{j i}=0$ is equivalent to the fact that the vector field $w_{k}$ is a covariant almost analytic one. Applying $\nabla^{j}$ to (4.10), we obtain

$$
\nabla^{j} \boldsymbol{t}_{j k}=\left(\nabla^{j} \boldsymbol{\phi}_{j}{ }^{r}\right)\left(\nabla_{r} \boldsymbol{w}^{l}\right) \boldsymbol{\phi}_{l k}+\boldsymbol{\phi}_{j}{ }^{r}\left(\nabla^{j} \nabla_{r} \boldsymbol{w}^{l}\right) \boldsymbol{\phi}_{l k}+\boldsymbol{\phi}_{j}{ }^{r}\left(\nabla_{r} \boldsymbol{w}^{l}\right) \nabla^{j} \boldsymbol{\phi}_{l k}-\nabla^{j} \nabla_{j} \boldsymbol{w}_{k} .
$$

Making use of (4.1) and (4.3), the last equation becomes

$$
\nabla^{j} t_{j k}=-\left\{\nabla^{r} \nabla_{r} w_{k}-R^{*}{ }_{k r} w^{r}+N(w)_{k}\right\} .
$$

In the next place, we shall compute

$$
\nabla^{j}\left(t_{j k} w^{k}\right)=\left(\nabla^{j} t_{j k}\right) w^{k}+t_{j k} \nabla^{j} w^{k}
$$


Substituting (4.10) and (4.11) into the equation above, we have

$$
\begin{aligned}
\nabla^{j}\left(t_{j k} w^{k}\right)= & -\left\{\nabla^{r} \nabla_{r} w_{k}-R_{k r}^{*} w^{r}+N(w)_{k}\right\} w^{k} \\
& +\phi^{l j} \phi^{k i}\left(\nabla_{\iota} w_{k}\right) \nabla_{j} w_{i}+\left(\nabla_{j} w_{i}\right) \nabla^{j} w^{i} .
\end{aligned}
$$

Now, if we put $t^{2}(w)=t_{j k} t^{j k}$, then

$$
t^{2}=2\left\{\left(\nabla_{j} w_{k}\right) \nabla^{j} w^{k}+\phi^{l j} \phi^{k i}\left(\nabla_{\imath} w_{k}\right) \nabla_{j} w_{i}\right\}
$$

Consequently, from (4.12) and (4.13), we have

$$
\nabla^{j}\left(t_{j k} w^{k}\right)+\frac{1}{2} t^{2}=-\left\{\nabla^{r} \nabla_{r} w_{k}-R_{k r}^{*} w^{r}+N(w)_{k}\right\} w^{k} .
$$

If $M$ is compact, by integration of the last equation, we have the following

THEOREM 4.2. In a compact *O-space $M$, the integral formula

$$
\int_{M}\left[\left(\nabla^{i} \nabla_{j} w_{i}-R^{*_{i r}} w^{r}+N(w)_{i}\right) w^{i}+\frac{1}{2} t^{2}\right] d \sigma=0
$$

is valid for any vector field $w_{i}$.

We have seen that a covariant almost analytic vector field $w_{i}$ satisfies the equation (4.9). Conversely, consider a vector field $w_{i}$ satisfying (4.9). Then if $M$ is compact, we have $t^{2}=0$ i.e. $t_{j i}=0$ by virtue of (4.14), so $w_{i}$ is covariant almost analytic. Thus we have

THEOREM 4.3. A necessary and sufficient condition for a vector field $w_{i}$ in a compact ${ }^{*} O$-space to be a covariant almost analytic vector field of the first kind is that the equation (4.9) is satisfied.

In general, $\widetilde{w}^{i}=\varphi_{r}{ }^{i} w^{r}$ is not necessarily a contravariant almost analytic vector field even though $w^{i}$ is a contravariant almost analytic one, but about covariant almost analytic vector fields we have the following

THEOREM 4.4. In an $* O$-space, if a vector field $w_{i}$ is a covariant almost analytic vector field of the first kind, then $\widetilde{w}_{i}=\phi_{i}{ }^{r} w_{r}$ is also covariant almost analytic one of the same kind.

PROOF. 


$$
\begin{aligned}
& \boldsymbol{\varphi}_{j}{ }^{r} \nabla_{r} \widetilde{\boldsymbol{w}}_{i}-\boldsymbol{\phi}_{i}{ }^{r} \nabla_{j} \widetilde{\boldsymbol{w}}_{r}=\left\{\boldsymbol{\varphi}_{j}{ }^{r}\left(\nabla_{r} \boldsymbol{\phi}_{i s}\right)-\boldsymbol{\phi}_{i}{ }^{r}\left(\nabla_{j} \boldsymbol{\phi}_{r s}\right)\right\} \boldsymbol{w}^{s}+\boldsymbol{\phi}_{j}{ }^{r} \boldsymbol{\phi}_{i}{ }^{s} \nabla_{r} \boldsymbol{w}_{s}+\nabla_{j} \boldsymbol{w}_{i} \\
& =\varphi_{j}{ }^{t}\left(* O_{t i}^{k r} \nabla_{k} \varphi_{r s}\right) w^{s}+* O_{j i}^{r s} \nabla_{r} w_{s} \text {. }
\end{aligned}
$$

From (4.6), we have

$$
\nabla_{j} w_{i}+\varphi_{j}^{r} \phi_{i}^{s} \nabla_{r} w_{s}=0 \text { i.e. } * O_{j i}^{r s} \nabla_{r} w_{s}=0
$$

By (4.1) and (4.15), we have

$$
\varphi_{j}^{r} \nabla_{r} \widetilde{w}_{i}-\phi_{i}^{r} \nabla_{j} \widetilde{w}_{r}=0
$$

from which the Theorem follows.

Moreover we can easily see that in an $* O$-space a covariant almost analytic vector field $w_{i}$ is an imcompressible vector field. In fact, transvecting $f^{i j}$ to (4.15), we get $\nabla^{i} w_{i}=0$.

\section{BIBLIOGRAPHY}

[1] S. Helgason, Differential geometry and symmetric spaces, Academic press, 1962.

[2] C. T. Hsu, On some structures which are similar to the quaternion structure, Tòhoku Math. Journ., 12(1960), 403-428.

[3] Y. Mutô, On some almost Kählerian spaces, Tôhoku Math. Journ., 14(1962), 344-364.

[4] S. SASAKI, On the differential geometry of tangent bundles of Riemannian manifolds, Tôhoku Math. Journ., 10(1958), 338-354.

[5] S. SASAKI, Homogeneous contact transformations, Tôhoku Math. Journ., 14(1962), 369397.

[6] S. TAChibana, On almost analytic vectors in almost Kählerian manifolds, Tôhoku Math. Journ., 11(1959), 247-265.

[ 7 ] S. TACHIBANA, On almost analytic vectors in certain almost Hermitian manifolds, Tôhoku Math. Journ., 11(1959), 351-363.

[8] P. TONDEUR, Structure presque kählerienne naturalle sur le fibre des vecteurs covariants d'une variété riemannienne, C. R. Acad. Sci. Paris, 254(1962), 407-408.

[9] K. YANO, The theory of Lie derivatives and its applications, Amsterdam, 1955.

[10] K. YANo AND M. AKo, Almost analytic vectors in almost complex spaces, Tôhoku Math. Journ., 13(1961), 24-45.

YAMAGATA UNIVERSITY. 